\title{
Design and Analysis of a Compliant Bimanual Rehabilitation Device
}

\author{
Samuel McAmis and Kyle B. Reed \\ Department of Mechanical Engineering \\ University of South Florida \\ Tampa, Florida, 33620 \\ Email: smcamis@mail.usf.edu, kylereed@usf.edu
}

\begin{abstract}
Bimanual rehabilitation allows an individual with hemiparesis to use their sound arm to help rehabilitate their impaired arm and shows promise as a means of low cost home use rehabilitation. However, few studies have attempted to determine the best combination of bimanual symmetry modes and coupling stiffnesses. To examine the effects of stiffness and symmetry type, we have developed a compliant bimanual rehabilitation device (CBRD) that allows the two hands to be coupled in a variety of symmetry modes and coupling stiffnesses so that their efficacy may be tested. Preliminary analysis has shown that the CBRD can couple the motions of two healthy individuals, which we are using to simulate an individual with a hemiparesis, and improves bimanual task performance of a single individual. The results also show that a higher coupling stiffness reduces the coupling error and that some tasks were easier to complete in visual symmetry.
\end{abstract}

\section{INTRODUCTION}

Bimanual rehabilitation allows an individual to selfrehabilitate by guiding his paretic arm with his sound arm using an external physical coupling. This coupling allows the individual to move his impaired hand through motions he would not otherwise be able to make while still giving him complete control over the motion generated, something that a physical therapist or robot would not be able do. This method also allows for upper-limb rehabilitation devices that are significantly lower in cost than robotic systems since much of the required force could be provided by the patient's healthy limb instead of the larger motors included on many current upper-limb rehabilitation robots. This would result in a lower cost and safer rehabilitation method that could be used at home, increasing access to rehabilitation. Here, we present a device that allows the hands to be coupled in several common symmetry modes and with a selectable coupling stiffnesses. The device was tested with healthy subjects in tasks that mimic aspects of hemiparesis as well as standard bimanual tasks.

\section{BACKGROUND}

The goal of upper-limb rehabilitation following a stroke is to enable a person to use both hands in activities of daily living. Of the new rehabilitation methods proposed and tested in recent years, many show positive results, but there is no method that clearly shows better results than traditional methods [1][2]. A common thread among the successful studies is that the amount of time spent training the affected arm plays an important role in improving the functional ability of the affected arm. As it is difficult for therapists to devote as much time as is needed, researchers have looked to robotic and other methods to supplement rehabilitation.

\section{A. Traditional and Robotic Rehabilitation Techniques}

Conventional stroke rehabilitation therapies, such as the Bobath method [3] and proprioceptive neuromuscular facilitation [4] have been used for decades. However, these methods are time-consuming and require significant effort from physical therapists. Forced use [5] and the more recently developed Constraint-Induced Movement Therapy [6] bind the sound arm and force the individual to use only the paretic limb, but is only viable for small to moderate impairment.

In recent years, robotic technologies have been used to provide rehabilitation to individuals, allowing access to rehabilitation for longer and more frequent periods of time. However, recent review papers have stated that it is unclear whether robotic methods have the potential to produce greater benefits than conventional techniques when practiced for the same amount of time [1][2].

To allow patients greater access to rehabilitative training, several methods have been developed to allow patients to rehabilitate at home [7][8]. However, many of these homebased methods use a home computer with limited accessories that cannot provide assistance forces and can only operate over a small workspace. These methods are able to provide some benefit, but the rehabilitation effect is limited to people who have relatively high motor function.

\section{B. Bimanual Rehabilitation}

The idea behind bimanual rehabilitation is that an individual uses their healthy arm to assist their own impaired arm through simultaneous bimanual motions. The key mechanism of rehabilitation is that the same neural signal is sent to the arms, which results in the same proprioceptive feedback from each limb since the arms are constrained to move together. Sending the same efferent signals to each limb will result in similar afferent signals from the limbs, which will help retrain the motor pathways to the impaired side [9][10]. Several research groups have studied certain aspects of coupled and uncoupled bimanual rehabilitation [11][12][13][14][15], but few studies to date have examined what the ideal physical parameters for bimanual interaction should be.

The above studies either did not physically connect the hands or coupled the hands rigidly, and few studies have analyzed the effect of the coupling stiffness. The ideal coupling is likely an intermediate stiffness, since a soft coupling would prevent severely impaired individuals from using this training method and, with a completely rigid connection, the individual is likely to apply minimal force in their impaired hand since the healthy side will dictate all the motions [1][16].

It is not currently known which types of symmetry modes are most effective for bimanual rehabilitation. Mirror motions have been the most commonly used in bimanual rehabilitation studies to date. However, most daily tasks occur in a visual reference frame where the hands move in the same direction. Three common reference frames used in bimanual rehabliltation are Mirror or Joint Space Symmetry (JSS), Visual Symmetry (VS), and Point Mirror Symmetry (PMS), as shown in Fig. 1 [17][18]. 


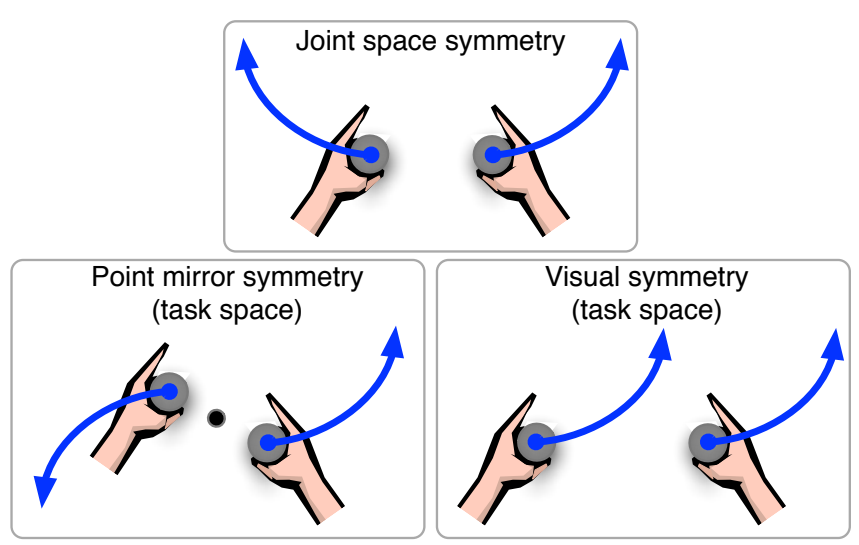

Fig. 1. Common bimanual symmetry modes include Joint Space Symmetry (JSS) where the joint angles are mirrored, Visual Symmetry (VS) where the hands move through the same visual path, and Point Mirror Symmetry (PMS) where the hand motions are mirrored about a point in space.

Preliminary studies of bimanual symmetric motions on healthy participants have shown that it is easier to follow and recreate motions in VS and JSS than in PMS [19] and that a coupling stiffness of $200 \mathrm{~N} / \mathrm{m}$ or greater resulted in better path following and motion coupling. These studies were performed on a pair of Phantom Omni force feedback devices. The research presented here details the design and preliminary analysis of a device that will allow testing the efficacy of different coupling stiffnesses and symmetry modes in bimanual rehabilitation.

\section{Compliant Bimanual Rehabilitiation Device}

We developed a device that physically couples two handles in a selected symmetry with an adjustable coupling stiffness, shown in Fig. 2. It is divided into two sub-assemblies: the coupling system connects the handle in a desired symmetry mode; the compliant handle assemblies allow the handles to be moved away from the correct symmetric positions, but provide a spring force back towards the symmetric positions. The total material cost to produce the prototype, including sensors and interface hardware, was approximately $\$ 700$.

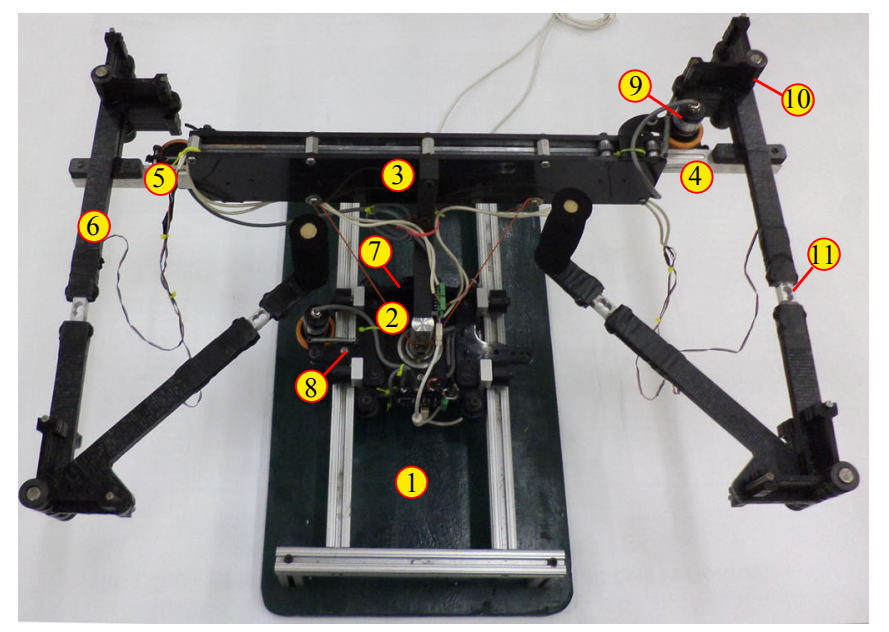

Fig. 2. CBRD components: (1) Base, (2) Carrier Assembly, (3) Upper Assembly, (4) Right Handle Slide, (5) Left Handle Slide, (6) Compliant Handle Assembly, (7) Lock for JSS and VS modes, (8) Lock for PMS mode, (9) Rotary Encoder, (10) Spring Stack, (11) Load Cell.

\section{A. Coupling System}

The coupling system consists of a four jointed mechanism with three prismatic joints and one revolute joint. The first joint, hereafter referred to as the Y-axis joint, is prismatic and connects the base to a captive carrier assembly that supports the rest of the device, allowing for motion towards or away from the participant for both JSS and VS modes. A bolt with a captive nut is used to remove this degree of freedom for PMS. The second joint, in the center of the carrier assembly, is revolute and connects the carrier assembly to the upper assembly and allows the latter to rotate for PMS. This joint is referred to as the Z-axis joint. A locking plate removes this degree of freedom for JSS and VS symmetry modes.

The remaining two joints allow for lateral motion of both handle slides in JSS and VS and for radial motion in PMS. The motion of these $\mathrm{X}$-axis joints is monitored by optical encoders with a resolution of $0.25^{\circ}$. The encoders contact the handle slides with friction wheels of radius $2.38 \mathrm{~mm}$, resulting in a linear resolution of $0.10 \mathrm{~mm}$.

The motions of the last two joints are coupled by cable runs on the back side of the upper assembly. As shown in Fig. 3, altering the cable path changes the coupling. If the cables loop around the pulleys an odd number of times, the motions of the handle slides will be mirrored, as necessary for JSS and PMS. If the cable loops around the pulleys an even number of times, the handle slides will move in the same absolute direction as required for VS.

In JSS and VS, each handle has a workspace $330 \mathrm{~mm}$ deep and $431 \mathrm{~mm}$ wide, starting $124 \mathrm{~mm}$ from the centerline. In VS the distance between the handles is $679 \mathrm{~mm}$, so that the maximum extension for one handle is the minimum extension for the other. In PMS the workspace is a disk with an inner radius of $124 \mathrm{~mm}$ and an outer radius of $555 \mathrm{~mm}$. At full extension in JSS or PMS, the handles are $1110 \mathrm{~mm}$ apart.

The stiction in the base to carrier assembly joint is approximately $4-20 \mathrm{~N}$, though typically less than $10 \mathrm{~N}$, dependent on the extension of the handle slides and the resultant torque applied to the joint. The resistance in the carrier plate-upper assembly joint is negligible. The stiction in the upper assembly to handle slide joint is approximately 10$15 \mathrm{~N}$. The total mass of the carrier and all moving components is $6.9 \mathrm{~kg}$. Future versions will reduce the stiction and weight.

\section{B. Compliant Handle Assembly}

Each handle is connected to the coupling system by a compliant assembly that forces the handle towards the correct position, but allows the handle to deviate from it. The compliant handle assembly consists of three links, connected by two pins, and a stack of custom torsion springs on each pin. These springs consist of an L-shaped piece of acetal plastic, $51 \mathrm{~mm}$ per leg, with a hole for the connecting pin where the

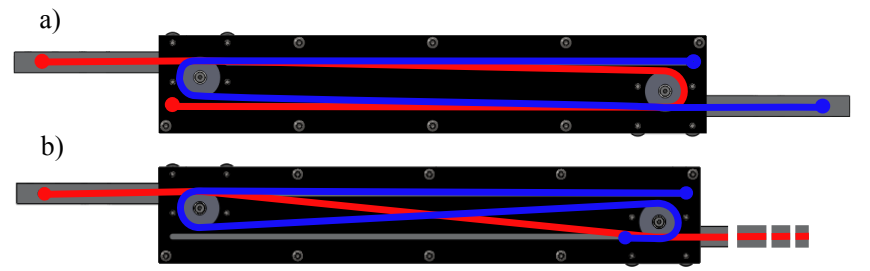

Fig. 3. Diagram of cable layouts as viewed from the rear: a) Joint Space Symmetry and Point Mirror Symmetry, b) Visual Symmetry 
legs meet. We use custom springs because standard torsion springs are designed for larger deflections than we are using, and to achieve the same stiffness, standard springs require more material, substantially increasing the size and weight. These custom springs also allow for more control over the stiffnesses implemented. The performance of the custom springs was confirmed to be linear over the range used.

In the assembly, the second and third links make up the hypotenuse and one leg, respectively of a $45^{\circ}-45^{\circ}-90^{\circ}$ triangle, with the handle at the $90^{\circ}$ corner. This results in the torques about the pins producing a symmetric stiffness ellipse at the handle, for small deflections, although large deflections will result in distorted stiffness ellipse. Future versions will optimize the shape of the stiffness elipse.

The compliant handle assembly is designed for a maximum deflection of $75 \mathrm{~mm}$ in any direction. For this deflection, the maximum width of the springs is $6 \mathrm{~mm}$, hence a stack of $6.4 \mathrm{~mm}$ thick springs is used to achieve higher stiffnesses. Each spring adds $110 \mathrm{~N} / \mathrm{m}$ to the stiffness of the connection between the handle and the handle slide, however, since both handles are connected in this way, the overall coupling stiffness added by each set of springs is $55 \mathrm{~N} / \mathrm{m}$, and the maximum combined deflection from correct coupled positions is $150 \mathrm{~mm}$. The stiffness ellipse for one handle with two springs is shown in Fig. 4.

The forces in the links are monitored by shear load cells. From the load cell readings, the force on the handle can be calculated, and given a known joint stiffness, based on the number of springs used, the joint deflection can be calculated, along with the handle position.

\section{Display and Interaction Game}

The workspace of the device is visually represented on a display located above and slightly behind the device to allow users to interact with visually displayed targets. The displayed workspace was scaled down by a factor of $2.5: 1$, resulting in a visual workspace area $132 \mathrm{~mm}$ tall and $442 \mathrm{~mm}$ wide. For consistency, unless otherwise noted, all dimensions given are for the physical workspace. Desired positions of the handles were presented as red circles $40 \mathrm{~mm}$ (16 mm displayed) in diameter. The right and left handles were displayed as green and blue circles, respectively, both $40 \mathrm{~mm}$ in diameter.

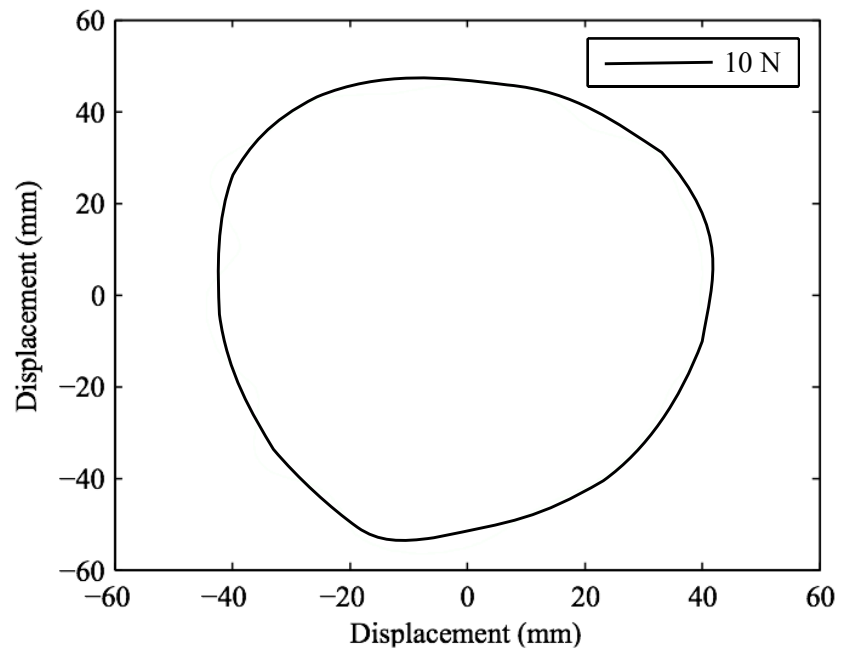

Fig. 4. Diagram of cable layouts as viewed from the rear: a) Joint Space Symmetry and Point Mirror Symmetry, b) Visual Symmetry
For all of the studies presented here, the task that participants were asked to complete consisted of matching the handle position(s) with the desired position(s). Each trial consisted of a series of eighteen segments, beginning with the display of randomly generated desired positions. The segment would end and, after a brief delay, the desired position would shift to a new position once the handle position was within $5 \mathrm{~mm}$ of the desired position, or 15 seconds had elapsed since the desired position was first displayed.

\section{PROCEDURE}

To evaluate the effectiveness of the device at coupling hand motions, a series of studies were conducted. The eventual goal is stroke rehabilitation; here, we are using two people to mimic the lack of bimanual coordination that occurs in individuals with stroke. This is a harsher test since the two participants are completely uncoupled neurally whereas an individual with stroke can couple the motions, but cannot fully control one of the arms. Thus, we evaluated the device in both a dual and single participant study.

\section{A. Two Participant Study}

In the dual-participant study, two participants stood in front of the device and each grasped a handle. The participant on the right held the right handle and the participant on the left held the left handle, mimicking the way that it would be held by a person with a stroke during rehabilitation. For each trial, one participant was designated as the guiding participant and the other participant was considered the following participant. The desired positions and handle positions were only displayed to the guiding participant and the following participant was asked to close their eyes or use a blindfold. A curtain separated the participants so that the guiding participant could only see their side of the device and the computer screen. The purpose of the two participant study was to quantify the performance of the device when one hand applies minimal input to the system.

The participants were asked to complete two types of tasks in different coupling symmetry modes and with different coupling stiffnesses. The symmetry modes tested were JSS and VS; PMS was omitted because it has been shown to be more difficult to coordinate bimanual motions in [19] and to limit the total study time to $1 \mathrm{hr}$ to reduce the possibility of participant fatigue. The coupling stiffnesses tested were $110 \mathrm{~N} / \mathrm{m}$ and $380 \mathrm{~N} / \mathrm{m}$. The lower stiffness was selected to be between $50 \mathrm{~N} / \mathrm{m}$ and $200 \mathrm{~N} / \mathrm{m}$ since this was shown to be an area of transition in path perception accuracy [19]. The $380 \mathrm{~N} / \mathrm{m}$ stiffness was selected as the highest possible stiffness without reducing the compliant workspace area below the maximum diameter of $300 \mathrm{~mm}$.

In one task, hereafter referred to as Two Person-Guiding Visible (2P-GV), only the guiding participant's desired and handle position were displayed. For this task, the guiding participant was asked to match their handle position with the desired position as quickly as possible. In the other task, hereafter referred to as Two Person-Following Visible (2P-FV), the following participant's desired position and both handle positions were displayed. For this task, the guiding participant was asked to match the following participant's handle position with the desired position.

Both participants completed all combinations of symmetry mode, stiffness and task type twice, once as the guide and once as the follower. The overall order of symmetry mode, 
stiffness, task and guiding participant was randomized for each pair of participants. However, to avoid confusion, and reduce delay time from switching configurations, the trials for each coupling stiffness were presented together. Similarly, for each coupling stiffness, all of the trials for one symmetry mode were presented before changing the symmetry mode, and for each symmetry mode, one guiding participant completed both tasks before the guiding participant was changed. Ten participants performed this study with IRB approval: eight were male, all were right handed, age 21-61 years old.

\section{B. Single Participant Study}

In this study, a single participant stood in front of the device and held both handles. The participants were asked to complete three types of tasks in different coupling symmetry modes and with the handles of the device in one of two coupling conditions: either physically coupled in the desired symmetry mode, or uncoupled. The symmetry modes tested were the same as those tested in the two participant study. When the handles were coupled, a coupling stiffness of $380 \mathrm{~N} / \mathrm{m}$ was used for consistency with the two participant study.

For the physically coupled trials, the device was locked in the desired symmetry mode. To uncouple the handles, neither the $\mathrm{Y}$ nor $\mathrm{Z}$-axis joints were locked, allowing the handles to be positioned independently, anywhere in the device workspace, however, they were dynamically coupled by inertia and friction, and the handles would still twist by the same angle about the the $\mathrm{Z}$-axis. In the uncoupled trials, participants were instructed to couple their hand motions in the desired symmetry mode.

One task was identical to that of the two participant study. In this task, referred to as One Person-Single Visible (1P-SV), participants were asked to match one handle position to a desired position as quickly as possible, while moving both of their hands together in the desired symmetry mode. In another task, referred to as One Person-Both Visible (1P-BV), both left and right handle and desired positions were displayed in the current symmetry mode, and participants were asked to match both handle positions to the desired positions. The purpose of these tasks was to analyze the effect of the CBRD on assisting a healthy paricipant in coordinating their hand motions.

In the third task, referred to as One Person-Distorted Positions (1P-DP), both left and right handle and desired positions were displayed, but their positions from the zero position for the symmetry mode were distorted by a factor of $1: 1.5$, and participants were, again, asked to match both handle positions to the desired positions. The purpose of this task was to mimic the decreased perceptional ability of individuals with stroke and test the device's ability to transmit forces.

Participants completed all combinations of symmetry mode, coupling condition and task twice; 1P-SV was completed once with the left visible and once with the right visible, and similarly 1P-DP was completed once with the distortion on the left and once with the distortion on the right. The 1P-BV condition was simply completed twice under the same conditions.

The overall order of symmetry mode, coupling condition, task, and left or right display/distortion was randomized. However, to avoid confusion, and reduce delay time from switching configurations, the trials for each symmetry mode were presented together. Similarly, for each symmetry mode, all of the trials for one coupling condition were presented before changing the coupling condition. If the first trial that a participant would conduct in a new symmetry mode was uncoupled, and only one desired position displayed, i.e. they would have neither visual nor haptic indication of how to couple their hand motions, they were permitted to practice moving in the desired symmetry mode until they understood the correct way to couple their motions. Six participants performed this study with IRB approval, five were male, all were right handed, age 21-25.

\section{Analysis}

To quantify performance during a trial, the average completion time and the average coupled position error were analyzed. The average completion time for a trial was determined by calculating the average segment time, from the display of a desired position or positions to the matching of the handle position(s) with the desired position(s), and averaging these segment times for each trial. The average coupled position error was the average, for a trial, of the distance between the right handle position and the projected symmetric position of the left handle at the end of each segment. The projected symmetric position of the left handle was determined by mirroring the position of the handle for JSS mode or adding $679 \mathrm{~mm}$ to the left handle position for VS mode.

For statistical analysis, we conducted an analysis of variance (ANOVA) to analyze the effects of symmetry mode, coupling stiffness or condition, task type and guiding side on the average completion time and average coupling position error. When the ANOVA yielded significant results, we used Tukey's honestly significant difference test. We used an alpha of 0.05 for all statistical tests

\section{A. Two Participant Study}

\section{RESUlTS}

Since the two types of tasks in the dual-participant study are inherently different: moving a handle directly vs. moving a handle through the coupling of the device, we performed our analysis with both task types together, and for each task type individually.

For both tasks, an analysis of the average completion time showed statistically significant results between symmetry modes $\left(\mathrm{F}_{1,79}=9.31, \mathrm{p}=0.003\right)$, coupling stiffnesses $\left(\mathrm{F}_{1}, 79=4.69, \mathrm{p}=0.03\right)$ and task types $\left(\mathrm{F}_{1}, 79=131.2, \mathrm{p}<0.001\right)$. Post hoc analysis showed that the completion time was lower for VS mode, for the $110 \mathrm{~N} / \mathrm{m}$ stiffness, and for the $2 \mathrm{P}-\mathrm{GV}$ task. The completion times for the symmetry modes and tasks are shown in Fig. 5. The average completion time for $2 \mathrm{P}-\mathrm{GV}$ was $2.7 \mathrm{~s}$, and the average completion time for $2 \mathrm{P}-\mathrm{FV}$ was $5.7 \mathrm{~s}$.

For the $2 \mathrm{P}-\mathrm{GV}$ task, analysis of the average completion time did not show statistically significant results between symmetry modes or coupling stiffnesses. For the 2P-FV task, analysis of the average completion time showed statistically significant results between symmetry modes $\left(\mathrm{F}_{1}, 39=9.45, \mathrm{p}=0.004\right)$. Post hoc analysis showed that the average completion time was lower for VS than for JSS.

For both tasks, analysis of the average coupled position error showed statistically significant results between symmetry modes $\left(\mathrm{F}_{1}, 79=4.90, \mathrm{p}=0.03\right)$ and coupling stiffnesses $\left(\mathrm{F}_{1}, 79=265.48, \mathrm{p}<0.001\right)$. Post hoc analysis showed that the the error was smaller for JSS than VS, $51 \mathrm{~mm}$ and $56 \mathrm{~mm}$, 
respectively, and that the error was lower for the $380 \mathrm{~N} / \mathrm{m}$ stiffness than for the $110 \mathrm{~N} / \mathrm{m}$ stiffness.

For the $2 \mathrm{P}-\mathrm{GV}$ task, analysis of the average coupled position error showed statistically significant results between coupling stiffnesses $\left(\mathrm{F}_{1}, 39=140.53, \mathrm{p}<0.001\right)$. For the $2 \mathrm{P}-\mathrm{FV}$ task, analysis of the coupled position error showed statistically significant results between coupling stiffnesses $\left(\mathrm{F}_{1}, 39=117.97, \mathrm{p}<0.001\right)$. Post hoc analysis showed that the average error was lower for the $380 \mathrm{~N} / \mathrm{m}$ stiffness and was comparable to the average for both tasks.

\section{B. Single Participant Study}

For the single participant study, the analysis was performed both with the data from the three tasks combined as well as for the data of the tasks individually. The coupled position error was only analyzed for the 1P-SV task because in the other tasks, the correct final position for both handles was displayed

For all three tasks and both coupling conditions, analysis of the average completion time showed statistically significant results between the task types $\left(\mathrm{F}_{2}, 143=40.17, \mathrm{p}<0.001\right)$. Post hoc analysis showed that 1P-SV was completed faster than 1P-BV, which, in turn, was completed faster than 1P-DP. The average completion times for 1P-SV, 1P-BV, and 1P-DP were $2.2 \mathrm{~s}, 2.8 \mathrm{~s}$ and $3.3 \mathrm{~s}$, respectively.

For the 1P-SV task and both coupling conditions, analysis of the average completion time showed statistically significant results between coupling conditions $\left(\mathrm{F}_{1}, 47=40.17, \mathrm{p}=0.003\right)$. Post hoc analysis showed that the task was completed faster with the handles coupled (Fig. 6).

For the coupled 1P-SV task, analysis of the average completion time showed statistically significant results between symmetry modes $\left(\mathrm{F}_{1}, 23=7.14, \mathrm{p}=0.05\right)$. Post hoc analysis showed that the task was completed faster in VS than in JSS. For the uncoupled 1P-SV task, analysis of the average completion time did not show statistically significant results.

For the 1P-BV task and both coupling conditions, analysis of the average completion time showed statistically significant results between coupling conditions $\left(\mathrm{F}_{1}, 47=34.13, \mathrm{p}=0.001\right)$. Post hoc analysis showed that the task was completed faster when the handles were coupled (Fig. 6).

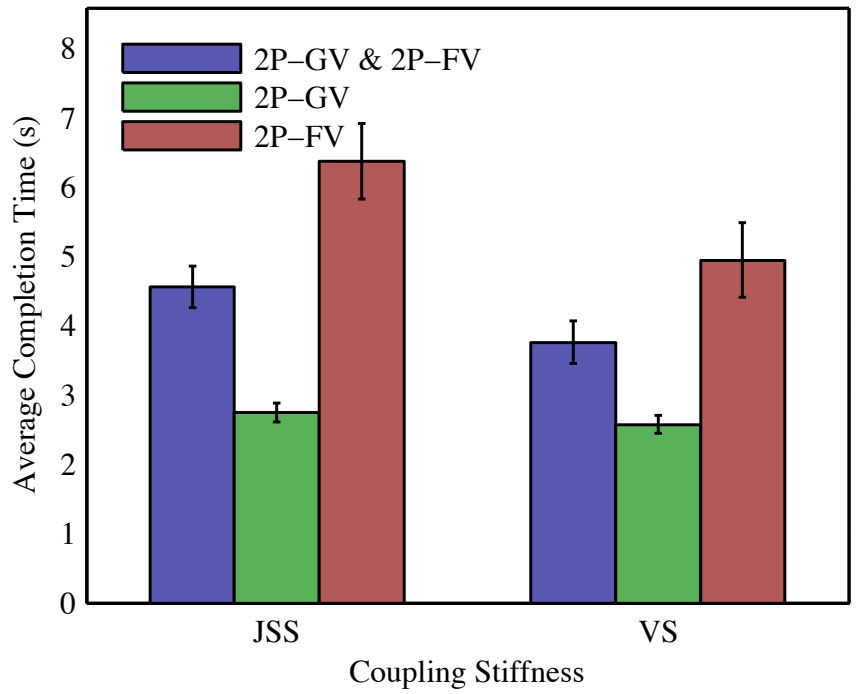

Fig. 5. Results of average completion time analysis for Two Participant Study. Error bars represent $95 \%$ confidence interval.
For the 1P-DP task and both coupling conditions, analysis of the average completion time showed statistically significant results between coupling conditions $\left(\mathrm{F}_{1}, 47=11.24, \mathrm{p}=0.002\right)$. Post hoc analysis showed that the task was completed faster when the handles were uncoupled (Fig. 6). Analysis of the completion time for the uncoupled 1P-DP task showed statistically significant differences between symmetry modes $\left(\mathrm{F}_{1}, 23=15.34, \mathrm{p}=0.001\right)$. Post hoc analysis showed that the task was completed faster in JSS than in VS. Analysis of the completion time for the coupled 1P-DP task did not show statistically significant results.

For the 1P-SV task and both coupling conditions, analysis of the coupled position error showed statistically significant results between symmetry modes $\left(\mathrm{F}_{1}, 47=8.7, \mathrm{p}=0.005\right)$ and coupling conditions $\left(\mathrm{F}_{1}, 47=32.2, \mathrm{p}<0.001\right)$. Post hoc analysis showed that the error was smaller in JSS than in VS, and when the handles were coupled.

For the coupled 1P-SV task, analysis of the coupled position error showed statistically significant results between symmetry modes $\left(\mathrm{F}_{1}, 23=45.54, \mathrm{p}<0.001\right)$. Post hoc analysis showed that the error was smaller for JSS than VS. For the uncoupled 1P-SV task, the error did not show statistically significant results between symmetry modes.

\section{DISCUSSION}

The two participant study showed that both the $380 \mathrm{~N} / \mathrm{m}$ stiffness and VS mode results in faster completion times. The higher stiffness may improve completion time due to better haptic communication with the following partner, but may also be attributable to better control over the dynamic motion of the system. The fact that $2 \mathrm{P}-\mathrm{FV}$ task is completed faster in VS than in JSS, as shown in Fig. 5, makes sense because in JSS the guiding participant must account for the mirrored motion of the handle that he is attempting to move to the desired position, while in VS the following handle moves in the same direction as the handle that he is controlling directly does. This indicates that for bimanual rehabilitation tasks in JSS mode, it may be beneficial to display the desired position of both handles so that an individual may focus on generating both motions together rather than on the motion of the healthy arm required to assist the impaired arm in the correct direction.

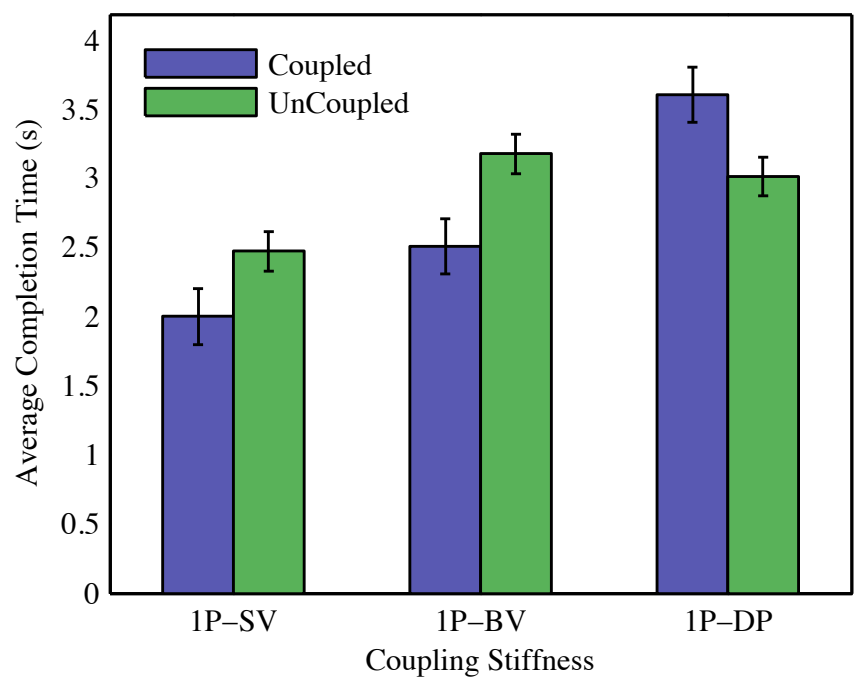

Fig. 6. Results of average completion time analysis for Single Participant Study. Error bars represent $95 \%$ confidence interval. 
The two participant study also showed that the coupled position error is smaller for the $380 \mathrm{~N} / \mathrm{m}$ stiffness than the $110 \mathrm{~N} / \mathrm{m}$ stiffness at approximately $30 \mathrm{~mm}$, and $75 \mathrm{~mm}$, respectively, corresponding to forces applied of $11.4 \mathrm{~N}$ and $8.25 \mathrm{~N}$, respectively, which is consistent with the friction in the coupling system as stated in Section III-A.

The coupled position error showed a difference between symmetry modes, indicating that there may be a difference in performance in coupling modes, although the difference is on the order of $10 \%$ of the coupled position error.

The 1P-SV task with the handles coupled showed that the average completion time was lower for VS than for JSS. This is consistent with the idea that many VS tasks, such as moving a large object, are done with the hands coupled together, and may be a more natural symmetry mode if only the desired position of one handle is displayed. However, our preliminary studies [19] show that uncoupled non-harmonic motions should also be faster in VS than in JSS. The difference may be attributable to friction and inertial forces slowing the motions enough to mask the differences in completion time. Therfore, further coupled bimanual studies on a device with lower impedance should be conducted, and an effort should be made to reduce the impedance of the CBRD.

For the 1P-DP task, the average completion time was lower when the handles were uncoupled. This makes sense because when the handles are coupled for this task, the participant must fight against the device to move the handles to the distorted desired positions. The forces required to reach the desired positions ranged from $0-45 \mathrm{~N}$.

The single participant study also showed that for the 1P-SV and 1P-BV tasks, when the handles were coupled in the desired symmetry mode, the average completion time was lower, as shown in Fig. 6. The figure also shows that the average completion time for 1P-SV uncoupled is comparable to 1P$\mathrm{BV}$ coupled, demonstrating that coupling motions through the CBRD can reduce the difficulty of matching two visually displayed positions to that of matching only one. These results show that coupling the hand motions through the CBRD improves performance of a healthy subject at completing bimanual tasks, indicating that it should be implemented in bimanual rehabilitation studies to test its efficacy.

\section{CONCLUSIONS AND FUTURE WORK}

Our results show that the CBRD effectively couples the bimanual motions of healthy subjects in JSS and VS modes, and that a higher coupling stiffness results in better performance in two participant bimanual tasks simulating hemiparesis. This two participant study also showed that when only the desired position of the following participant was displayed, the trials were completed faster in VS than JSS, and that displaying both desired positions in a JSS bimanual rehabilitation task may be beneficial.

Our future work includes making improvements to the CBRD to improve coupling performance and make it suitable for testing with individuals with stroke. These improvements include reducing the friction in the prismatic joints, optimizing the stiffness ellipse of the compliant handle assembly, and adding a dynamic arm rest with a means to secure an individual's impaired arm to the compliant handle assembly.

\section{REFERENCES}

[1] L. Marchal-Crespo and D. Reinkensmeyer, "Review of control strategies for robotic movement training after neurologic injury," Journal of NeuroEngineering and Rehabilitation, vol. 6, no. 1, p. 20, 2009.

[2] G. Kwakkel, B. J. Kollen, and H. I. Krebs, "Effects of Robot-Assisted Therapy on Upper Limb Recovery After Stroke: A Systematic Review," Neurorehabil Neural Repair, vol. 22, no. 2, pp. 111-121, 2008.

[3] B. Bobath, Adult hemiplegia: Evaluation and treatment. London, UK: Heinemann Medical Books Ltd., 1970.

[4] M. Knott and D. Voss, Proprioceptive Neuromuscular Facilitation: Patterns and Techniques, 2ed, 2nd ed. New York, NY: Harper \& Row Publishers Inc., 1968.

[5] R. Oden, "Systematic therapeutic exercises in the management of the paralyses in hemiplegia," JAMA, vol. 23, pp. 828-833, 1918.

[6] E. Taub, G. Uswatte, and R. Pidikiti, "Constraint-induced movement therapy: A new family of techniques with broad application to physical rehabilitation-a clinical review," Journal of Rehabilitation Res, vol. 36, no. 3, pp. 237-251, 1999.

[7] M. Johnson, X. Feng, L. Johnson, and J. Winters, "Potential of a suite of robot/computer-assisted motivating systems for personalized, home-based, stroke rehabilitation," Journal of NeuroEngineering and Rehabilitation, vol. 4, no. 1, p. 6, 2007.

[8] D. J. Reinkensmeyer, C. T. Pang, J. A. Nessler, and C. C. Painter, "Java therapy: Web-based robotic rehabilitation," Integration of Assistive Technology in the Information Age, vol. 9, pp. 66-71, 2001.

[9] C. Burgar, P. Lum, P. Shor, and H. Van der Loos, "Development of robots for rehabilitation therapy: The Palo Alto VA/Stanford experience," J. of Rehab Research and Development, vol. 37, pp. 663674, 2000.

[10] S. L. Wolf, D. E. LeCraw, and L. A. Barton, "Comparison of Motor Copy and Targeted Biofeedback Training Techniques for Restitution of Upper Extremity Function Among Patients with Neurologic Disorders," Physical Therapy, vol. 69, no. 9, pp. 719-735, 1989.

[11] P. Lum, D. Reinkensmeyer, R. Mahoney, W. Z. Rymer, and C. Burgar, "Robotic devices for movement therapy after stroke: Current status and challenges to clinical acceptance," Topics in Stroke Rehab, vol. 8, pp. 40-53, 2002.

[12] S. Hesse, G. Schulte-Tigges, M. Konrad, A. Bardeleben, and C. Werner, "Robot-assisted arm trainer for the passive and active practice of bilateral forearm and wrist movements in hemiparetic subjects," Archives of Physical Medicine and Rehab, vol. 84, no. 6, pp. 915 920, 2003.

[13] J. Whitall, S. Waller, K. Silver, and R. Macko, "Repetitive Bilateral Arm Training With Rhythmic Auditory Cueing Improves Motor Function in Chronic Hemiparetic Stroke," Stroke, vol. 31, no. 10, pp. 2390-2395, 2000.

[14] K. Jordan, M. Sampson, J. Hijmans, M. King, and L. Hale, "Imable system for upper limb stroke rehabilitation," in Virtual Rehabilitation (ICVR), 2011 International Conference on, june 2011, pp. 1 -2.

[15] S. Hesse, C. Werner, M. Pohl, J. Mehrholz, U. Puzich, and H. I. Krebs, "Mechanical arm trainer for the treatment of the severely affected arm after a stroke," Am J Phys Med Rehabil, vol. 87, pp. 779-788, 2008.

[16] R. A. Schmidt and R. A. Bjork, "New conceptualizations of practice: Common principles in three paradigms suggest new concepts for training," Psychological Science, vol. 3, no. 4, pp. 207-217, 1992.

[17] H. G. Malabet, R. A. Robles, and K. B. Reed, "Symmetric motions for bimanual rehabilitation," in Proc. IEEE/RSJ Int Intelligent Robots and Systems (IROS) Conf, 2010, pp. 5133-5138.

[18] S. McAmis and K. B. Reed, "Symmetry modes and stiffnesses for bimanual rehabilitation," in Proc. IEEE Int. Conf. Rehabilitation Robotics, 2011, pp. 1106-1111.

[19] S. H. L. McAmis and K. B. Reed, "Simultaneous perception of forces and motions using bimanual interactions," Haptics, IEEE Transactions on, vol. 5, no. 3, pp. 220-230, 2012. 